\title{
Sleep Disturbance and Its Association with Pain Severity and Multisite Pain: A Prospective 10.7-Year Study
}

Feng Pan (1) · Jing Tian · Flavia Cicuttini $\cdot$ Graeme Jones

Received: September 7, 2020 / Accepted: October 6, 2020 / Published online: October 21, 2020

(C) The Author(s) 2020

\begin{abstract}
Introduction: Sleep disturbance is often comorbid with chronic pain disorders, with emerging evidence suggesting a stronger effect of sleep disturbance on pain than vice versa; however, few studies have evaluated the longterm associations between sleep disturbance and pain. This study was to examine the associations of sleep disturbance with knee pain severity, number of painful sites (NPS) and persistent pain in a 10.7-year cohort study.

Methods: A total of 1099 community-dwelling older adults (age mean $\pm S D, 63 \pm 7.5$ years;
\end{abstract}

Electronic Supplementary Material The online version of this article (https://doi.org/10.1007/s40122020-00208-x) contains supplementary material, which is available to authorized users.

F. Pan $(\varangle) \cdot$ J. Tian · G. Jones

Menzies Institute for Medical Research, University of Tasmania, Private Bag 23, Hobart, TAS 7000,

Australia

e-mail: Feng.Pan@utas.edu.au

F. Cicuttini

Department of Epidemiology and Preventive

Medicine, Monash University Medical School,

Commercial Road, Melbourne, VIC 3181, Australia
$51 \%$ female) were recruited and followed up at 2.6, 5.1 and 10.7 years later. Data on demographics, body mass index, physical activity and comorbidities were collected. At each time point, sleep disturbance, knee pain severity and NPS were assessed by using questionnaires. Multisite pain (MSP) was defined as NPS $\geq 2$. Persistent knee pain or MSP was defined as having knee pain or MSP at all time points, respectively. Multivariable mixed-effects models and log-binomial regression were applied.

Results: In multivariable analyses, sleep disturbance was associated with greater knee pain severity ( $\beta$ 0.91/unit, 95\% CI $0.70-1.11$ ) and more NPS [(relative risk (RR) 1.10/unit, 95\% CI 1.07-1.14] in a dose-response manner. Persistent sleep disturbance was associated with persistent knee pain (RR 1.90, 1.26-2.87) and MSP (RR 1.29, 1.07-1.56). Persistent knee pain and MSP were also associated with persistent sleep disturbance (knee pain: $\mathrm{RR}=1.99$; $\mathrm{MSP}: \mathrm{RR}=$ 2.71 , both $P<0.05$ ).

Conclusions: Sleep disturbance was independently associated with greater pain severity and NPS in a dose-response manner. A reciprocal relationship between persistent sleep disturbance and persistent pain suggests treating either problem could help the other.

Keywords: Cohort study; Multisite pain; Musculoskeletal pain; Pain intensity; Sleep disturbance 


\section{Key Summary Points}

Why carry out this study?

Sleep problems are highly prevalent in patients with chronic pain conditions; the direction of causality remains unclear.

Evidence of the longitudinal relationships of sleep disturbance with pain severity and its distribution is lacking.

We sought to examine the long-term relationships between sleep disturbance and pain intensity and multisite pain, and the persistent impact of sleep disturbance on pain.

\section{What was learned from the study?}

We found that sleep disturbance was associated with greater pain severity and more painful sites. Further, there was a reciprocal relationship between persistent sleep disturbance and persistent pain.

The findings of this study highlight that treatment should target both sleep and pain in pain and sleep management in clinical practice, and that treating either problem could help the other.

\section{DIGITAL FEATURES}

This article is published with digital features, including a summary slide, to facilitate understanding of the article. To view digital features for this article go to https://doi.org/10.6084/ m9.figshare.13042151.

\section{INTRODUCTION}

Sleep problems including difficulties in falling or maintaining asleep and experiencing inadequate sleep are common in the general population with a prevalence of as high as 56\% [1-3]. Poor sleep has been associated with a range of detrimental health outcomes, such as cardiovascular disease, depression and mortality $[4,5]$. Musculoskeletal pain is another public health concern affecting $14-47 \%$ of the general population [6] and has a significant impact on individuals' physical function and quality of life. Conditions such as back pain, neck pain, and osteoarthritis typically represent musculoskeletal pain $[7,8]$. Knee pain is the most defining symptom of osteoarthritis of the knee that is the most frequently affected joint [9].

Sleep problems are highly prevalent in patients with chronic pain conditions; as many as $88 \%$ of patients complain of sleep disturbance [10]. The relationship between sleep problems and pain has been extensively investigated in prior cross-sectional studies. Traditionally, it has been thought that the two conditions are related reciprocally in a bidirectional manner [11]; however, there is accruing evidence suggesting that sleep problems are more significant predictors of pain than vice versa [12-14]. A recent systematic review and meta-analysis concluded that decline in sleep quality and quantity was linked to a two- to three-times risk of developing pain conditions [15]. Although underlying mechanisms of the sleep-pain link are not yet fully understood, neurobiological and inflammatory mechanisms have been suggested to be involved in the modulation of pain by sleep problems $[16,17]$.

Sleep disturbance has been found to be associated with the resolution and onset of chronic widespread pain defined on the basis of the American College of Rheumatology (ACR) definition [18-21]. However, it has been suggested that the ACR definition is too stringent to adequately address the impact of pain in multiple body sites (called multisite pain, MSP) that is more common and has more impact on physical and psychological health than singlesite pain [22-25]. To date, research on the 
longitudinal relationship between sleep and MSP is lacking. In addition, almost all prior studies investigating the relationship between sleep and pain used qualitative, not quantitative pain outcome measures and had not more than two sleep and pain assessments. This has thus limited the ability to explore the extent to which sleep influences pain intensity and the ability to address the long-term relationship between sleep pattern and persistent pain [15], which are of particular importance in preventing and managing both conditions. Therefore, the aim of this study was to examine the longitudinal associations of sleep disturbance with knee pain severity and MSP, and examine the relationship between sleep disturbance pattern and persistent knee pain and MSP among community-dwelling older people.

\section{METHODS}

\section{Participants}

This study was conducted as part of the Tasmanian Older Adult Cohort (TASOAC) study, which is a longitudinal and population-based cohort study conducted in Tasmania, Australia. The TASOAC study comprised individuals aged 50-80 years who were randomly selected from the electoral roll in Southern Tasmania $(N=229,000)$ using sex-stratified simple random sampling without replacement and three follow-ups were then conducted at a mean follow-up period of 2.6, 5.1 and 10.7 years. A total of 1100 were recruited into the study, and 875 , 768 and 563 were traced at the subsequent follow-ups, respectively. At each visit, participants underwent clinical assessments and completed questionnaires and general interview. This study was approved by the Southern Tasmanian Health and Medical Human Research Ethics Committee (Ref. no. H0006488), and written informed consent of all participants was obtained. This study was performed in accordance with the Helsinki Declaration of 1964 and its later amendments.

\section{Sleep Variables}

Participants were asked how they feel about their sleep during the last week using one item on a 4-point scale from the Assessment of Quality of Life (AQoL) questionnaire [26] at each time point with the following response options: (1) I am able to sleep without difficulty most of the time; (2) My sleep is interrupted some of the time, but I am usually able to go back to sleep without difficulty; (3) My sleep is interrupted most nights, but I am usually able to go back to sleep without difficulty; (4) I sleep in short bursts only. I am awake most of the night. We defined persistent sleep disturbance if participants reported either " 3 " or " 4 " at all time points.

\section{Pain Variables}

\section{Knee Pain Severity}

Knee pain severity was assessed using the Western Ontario and McMaster Universities Osteoarthritis Index (WOMAC) pain questionnaire at baseline and following three follow-ups. The WOMAC pain subscale consists of five questions which ask participants to rate their pain while walking, climbing stairs, sleeping at night, sitting/lying and standing. Each question is scored on a 10-point scale ranging from 0 to 9 , with " 0 " representing "no pain", and "9" representing "most severe pain". A total WOMAC score was calculated and scored ranging from 0 to 45 .

\section{Multisite Musculoskeletal Pain}

Musculoskeletal pain site was measured using a self-reported questionnaire at each time point, with the question on whether participants had pain (yes/no) in the seven sites (neck, back, hands, shoulders, hips, knees or feet). The total number of painful sites (NPS) was calculated by summing all sites with a range from 0 to 7 . MSP was defined as pain occurring in two or more sites [27]. Persistent pain in knees or MSP was defined if participants reported knee pain or MSP at all time points, respectively. 


\section{Knee Radiography}

A standing anteroposterior semiflexed radiograph of the right knee was obtained from participants at baseline. The radiograph was scored for osteophytes and joint space narrowing (JSN) on a $0-3$ point scale using the Altman atlas, as previously described [28]. Radiographic knee osteoarthritis (ROA) was defined if the knee had a score of one or greater for JSN or osteophytes.

\section{Covariates}

Participants' demographic factors were collected by interview and the questionnaires at baseline including age, sex, emotional problems, employment, and education level. Participants were asked "how much have you been bothered by emotional problems during the past 4 weeks, such as feeling anxious, depressed or irritable?" using one single item from the Short Form-8 [29]. Response options included "not at all", "very little", "moderately", "quite a lot" and "extremely". The presence of emotional problems was defined if participants gave a response of "very little" or more. Self-reported employment status was classified as "employed" if participants had a full/part-time job. Education attainment was classified into three categories: "low level" if participants completed school only; "medium level" if those had a trade/vocational certificate; and "high level" if participants had a university degree or above. Smoking history was defined if participants had ever smoked at least seven cigarettes, cigar or pipes every week for at least 3 months. Common conditions were collected including diabetes, heart attack, hypertension, thrombosis, asthma, bronchitis/emphysema, hyperthyroidism, hypothyroidism and rheumatoid arthritis. The presence of comorbidities was defined if participants had any comorbidities. Participants reported all prescribed medication, and any other over-the-counter medications they had taken in the last 2 weeks. Pain medications were extracted and dichotomised into whether they were used or not (yes/no). Body mass index (BMI) $\left(\mathrm{kg} / \mathrm{m}^{2}\right)$ was calculated on the basis of height and weight. As previously described [30], a pedometer (Omron HJ-003 and HJ-102, Omron Healthcare, Kyoto, Japan) was used to measure physical activity, expressed as steps/day. Participants were asked to wear a pedometer for seven consecutive days. Number of steps each day, the duration of wearing and type of physical activity were recorded. Considering the potential influence of seasonal variation, we measured participants' physical activity 6 months later. The average steps per day at both time points were calculated.

\section{Statistical Analysis}

ANOVA and ordinal $\chi^{2}$ test (Kruskal-Wallis test) were used in univariable comparisons of categorical and continuous variables, respectively. Linear and Poisson mixed-effects models with random intercept for participants were used to assess the potential associations of sleep with pain severity and NPS, respectively, before and after adjustment for covariates. The advantage of using mixed-effect models for multiple timepoint longitudinal data is that it can consider the dependence of repeated observations within participants and utilise all data including those who were lost to follow-up. Separate mixed-effect models with random intercept for participants and 'sleep $\times$ time' interaction term were performed to determine the temporal associations between sleep and pain. Log-binomial regression was used to examine the reciprocal relationship between persistent sleep disturbance and persistent pain in the knees and MSP. We used inverse probability weighting to determine whether our results were influenced by the participants who were excluded from the analyses because of loss to follow-up or missing data [31]. We also performed secondary analyses in those with ROA at baseline. Stata version 16 (StataCorp, USA) was used for all statistical analyses. Statistical significance was considered if a two-tailed level of $P$ value was less than 0.05 .

\section{RESULTS}

Of the 1100 participants who were recruited in the study, 1099 attended clinical assessments and completed general interview and 
questionnaires. Of those, 1008 had complete data on sleep, pain status and covariates at baseline. Among them, 818, 720 and 533 participants attended 2.6, 5.1 and 10.7-year followup assessments. Compared with participants who had complete data at 10.7-year follow-up, the rest of cohort appeared to be older, had a greater BMI, lower level of physical activity, more comorbidities and greater WOMAC pain score and NPS, and had a higher proportion of participants who took pain medication, being ever smoker and unemployed, and had more emotional problems and lower education level. No differences in sex, sleep disturbance and ROA were observed (data not shown). At baseline, the mean age of this sample was 62.9 (SD 7.4 ), and the sample included 51\% women. $38 \%$ reported no interrupted sleep and 60\% had ROA. The mean WOMAC pain score and NPS were 3.5 (SD 6.0) and 3.2 (SD 2.2), respectively; $45 \%$ and $72 \%$ of participants had pain in the knees and MSP. During three follow-ups, overall, the mean WOMAC pain, total NPS, the proportion of individuals having knee pain and MSP were consistent; and the proportion of individuals reporting sleep disturbance scores over time appeared relatively stable (Table S1).

Participants' characteristics at baseline according to sleep disturbance are shown in Table 1. Participants who reported worse sleep disturbance were more likely to be female, physically inactive, have comorbidities, take pain medications, and have emotional problems, unemployment and lower education level. WOMAC pain score, NPS and the proportion of participants having knee pain and MSP increased across sleep disturbance groups with the lowest score or percentage in those reporting no interrupted sleep. There were no differences in age, BMI, ROA and smoking history across the groups.

The relationships between sleep disturbance and WOMAC pain and NPS at each time point are displayed in Fig. 1. Pain severity and NPS increased with the extent of sleep disturbance (all $P$ for trend $<0.05$ ).

In univariable analyses, worse sleep disturbance was associated with a greater WOMAC pain score compared with those without sleep disturbance, as detailed in Table 2. Pain severity increased as the sleep disturbance got worse. After adjustment for age, sex, BMI, physical activity, comorbidities, pain medications, smoking history, emotional problems, employment, and education level, these associations remained statistically significant. Similarly, the extent of sleep disturbance was associated with greater NPS in a dose-response manner (Table 3). Participants reporting that their sleep was interrupted some of the time or worse had more than a $20 \%$ higher risk for having greater NPS relative to those reporting no sleep disturbance in univariable analyses (all $P<0.05$ ). The effect sizes were slightly attenuated after adjusting for covariates, but the results remained statistically significant. There was no significant interaction of sleep disturbance and time with pain.

The presence of persistent sleep disturbance was associated with persistent knee pain and MSP in univariable analyses in the whole population, as shown in Table 4. After adjustment for covariates, the sleep-pain associations were not largely changed. A reverse relationship was also observed: both persistent knee pain and MSP were also associated with persistent sleep disturbance. Furthermore, the results did not alter after using the inverse probability weighting (Table S2).

To further explore the relationships between sleep disturbance and pain in participants with ROA, we performed secondary analyses in those with ROA at baseline. We observed similar results as in the entire cohort; there was a dose-response relationship between sleep disturbance and pain severity and NPS in both univariable and multivariable analyses (Tables S3, S4). In addition, reciprocal associations between persistent sleep disturbance and persistent knee pain among participants with ROA in univariable analysis were observed, and the results became borderline significant after adjusting for covariates (Table 5).

\section{DISCUSSION}

This study is the first, to the best our knowledge, to evaluate the long-term relationships between sleep disturbance and pain, both of which had 
Table 1 Baseline characteristics of participants according to sleep disturbance

\begin{tabular}{|c|c|c|c|c|c|}
\hline & \multicolumn{4}{|l|}{ Sleep disturbance ${ }^{a}$} & \multirow[t]{2}{*}{$P$ value } \\
\hline & $1(n=386)$ & $2(n=284)$ & $3(n=270)$ & $4(n=68)$ & \\
\hline Age (years) & $62.9 \pm 7.3$ & $62.9 \pm 7.7$ & $63.1 \pm 7.5$ & $61.8 \pm 7.1$ & 0.611 \\
\hline Female (\%) & 47 & 52 & 56 & 51 & 0.044 \\
\hline Height $(\mathrm{cm})$ & $167.4 \pm 9.0$ & $167.5 \pm 9.1$ & $166.3 \pm 8.8$ & $166.8 \pm 9.1$ & 0.203 \\
\hline Weight $(\mathrm{kg})$ & $78.1 \pm 15.0$ & $77.8 \pm 14.8$ & $76.6 \pm 14.4$ & $81.4 \pm 17.9$ & 0.865 \\
\hline $\mathrm{BMI}\left(\mathrm{kg} / \mathrm{m}^{2}\right)$ & $27.8 \pm 4.7$ & $27.7 \pm 4.6$ & $27.7 \pm 4.7$ & $29.2 \pm 5.9$ & 0.319 \\
\hline PA (steps/day) & $8870.0 \pm 3262.2$ & $8550.6 \pm 3421.5$ & $8366.6 \pm 3237.9$ & $8318.8 \pm 3937.2$ & 0.044 \\
\hline Any comorbidities (\%) & 58 & 64 & 69 & 57 & 0.047 \\
\hline ROA (\%) & 56 & 64 & 61 & 68 & 0.055 \\
\hline Pain medications (\%) & 45 & 64 & 67 & 54 & $<0.001$ \\
\hline Ever smoking (\%) & 51 & 50 & 50 & 59 & 0.620 \\
\hline Emotional problems (\%) & 49 & 68 & 75 & 78 & $<0.001$ \\
\hline Employed (\%) & 44 & 39 & 36 & 34 & 0.022 \\
\hline \multicolumn{5}{|l|}{ Education level (\%) } & \multirow[t]{4}{*}{0.039} \\
\hline School only & 51 & 60 & 55 & 72 & \\
\hline Vocational training & 35 & 31 & 33 & 22 & \\
\hline University or higher & 14 & 9 & 12 & 6 & \\
\hline WOMAC pain $(0-45)$ & $2.0 \pm 3.9$ & $3.3 \pm 5.2$ & $4.8 \pm 6.8$ & $8.4 \pm 10.4$ & $<0.001$ \\
\hline Number of painful sites $(0-7)$ & $2.5 \pm 2.0$ & $3.2 \pm 2.1$ & $3.8 \pm 2.1$ & $4.2 \pm 2.2$ & $<0.001$ \\
\hline Knee pain (\%) & 34 & 48 & 55 & 56 & $<0.001$ \\
\hline Multisite pain (\%) & 60 & 77 & 82 & 85 & $<0.001$ \\
\hline \multicolumn{6}{|c|}{$\begin{array}{l}\text { Bold denotes statistically significant result. Values are the mean } \pm \mathrm{SD} \text { except for percentages; ANOVA and ordinal } \chi^{2} \text { test } \\
\text { (Kruskal-Wallis test) were used to test if there was a trend in the mean of each continuous and categorical variable across } \\
\text { sleep disturbance } \\
B M I \text { body mass index, } P A \text { physical activity, ROA radiographic knee OA, WOMAC Western Ontario and McMaster } \\
\text { Universities Osteoarthritis Index } \\
\text { a 4-point sleep disturbance scale: (1) I am able to sleep without difficulty most of the time; (2) My sleep is interrupted some } \\
\text { of the time, but I am usually able to go back to sleep without difficulty; (3) My sleep is interrupted most nights, but I am } \\
\text { usually able to go back to sleep without difficulty; (4) I sleep in short bursts only. I am awake most of the night }\end{array}$} \\
\hline
\end{tabular}

more than two assessments, and the persistent impact of sleep disturbance on pain. The results suggest that sleep disturbance was associated with greater pain severity and more painful sites in a population-based sample of those with and without ROA. There was a reciprocal relationship between persistent sleep disturbance and pain before and after adjustment for covariates, suggesting a mechanistic link between sleep disturbance and pain severity and its distribution.

The current study findings that the prevalence of sleep disturbance and musculoskeletal pain is high in our population were consistent 

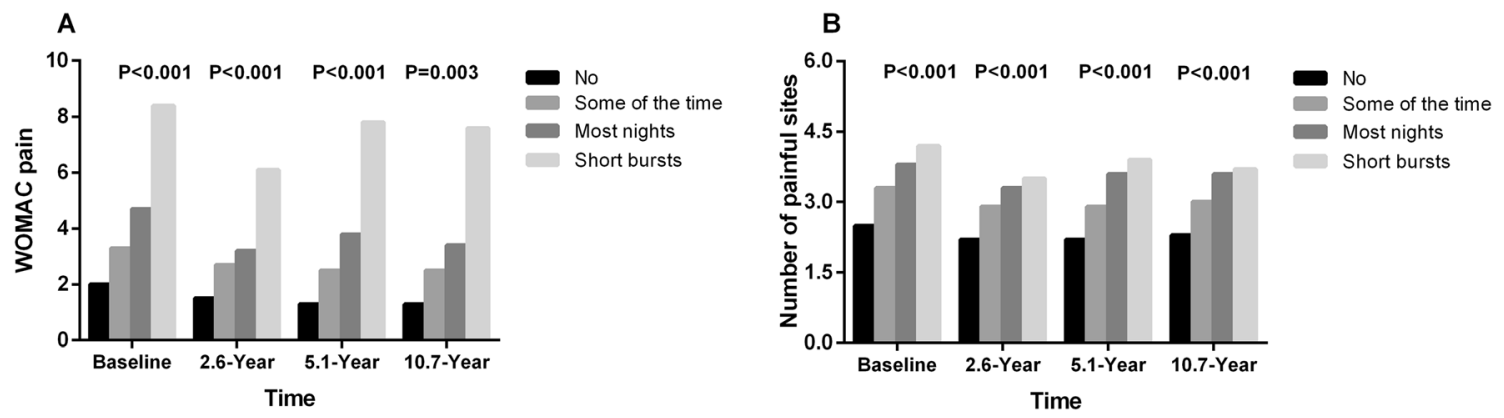

Fig. 1 Associations between sleep disturbance and pain severity and number of painful sites: a WOMAC pain; $\mathbf{b}$ number of painful sites. $P$ for trend determined by ANOVA test

Table 2 Association between sleep disturbance and WOMAC pain score over time using mixed-effects model in entire cohort

\begin{tabular}{|c|c|c|c|c|}
\hline \multirow[t]{2}{*}{ Sleep disturbance ${ }^{a}$} & \multicolumn{2}{|l|}{ Model 1} & \multicolumn{2}{|l|}{ Model 2} \\
\hline & $\bar{\beta}$ & $95 \% \mathrm{CI}$ & $\bar{\beta}$ & 95\% CI \\
\hline 1 & Reference & & Reference & \\
\hline 2 & 0.73 & $0.32,1.14$ & 0.48 & $0.07,0.89$ \\
\hline 3 & 1.54 & $1.07,2.01$ & 1.24 & $0.77,1.71$ \\
\hline 4 & 3.98 & $3.21,4.75$ & 3.84 & $3.07,4.62$ \\
\hline$P$ for trend & $<0.001$ & & $<0.001$ & \\
\hline
\end{tabular}

Bold denotes statistically significant result

a 4-point sleep disturbance scale: (1) I am able to sleep without difficulty most of the time; (2) My sleep is interrupted some of the time, but I am usually able to go back to sleep without difficulty; (3) My sleep is interrupted most nights, but I am usually able to go back to sleep without difficulty; (4) I sleep in short bursts only. I am awake most of the night

Model 1: Univariable analysis

Model 2: Adjusted for fixed factors (age, sex, body mass index, physical activity, comorbidities, pain medications, ever smoking, emotional problems, employment, and education level)

$\beta$ beta coefficient, $C I$ confidence interval

with previous studies. This corroborates the evidence that sleep disturbance [1-3] and pain occurring in multiple sites $[27,32]$ are common in the general population. A number of studies, to date, have investigated the sleep-pain link and reported that sleep disturbance is frequently associated with pain [14]. However, the pain was often assessed by non-quantitative questionnaires (yes/no) and evidence on how sleep disturbance patterns affect pain outcomes is lacking since few studies had sleep and pain assessments at more than two time points [15]. Therefore, our study not only further consolidates evidence that sleep disturbance is often comorbid with pain but also extends previous studies to show the associations between sleep and pain intensity and its distribution, and to demonstrate a reciprocal relationship between persistent sleep disturbance and pain outcome measures.

We found that sleep disturbance was associated with greater knee pain score. No study has investigated longitudinal associations between sleep disturbance and knee pain intensity, but this finding is indirectly supported by one previous clinical trial that has shown the efficacy of sleep intervention through cognitive behavioural therapy (CBT) on pain reduction over 
Table 3 Association between sleep disturbance and number of painful sites over time using mixed-effects model in entire cohort

\begin{tabular}{llllll}
\hline Sleep disturbance $^{\mathrm{a}}$ & \multicolumn{2}{l}{ Model 1 } & & \multicolumn{2}{l}{ Model 2 } \\
\cline { 2 - 2 } \cline { 5 - 5 } & RR & Refe CI & & RR & 95\% CI \\
\hline 1 & Refence & & Reference & \\
2 & 1.20 & $1.12,1.28$ & & 1.14 & $1.07,1.22$ \\
3 & 1.34 & $1.24,1.44$ & & 1.25 & $1.16,1.35$ \\
4 & 1.42 & $1.27,1.58$ & & 1.31 & $1.17,1.46$ \\
$P$ for trend & $<0.001$ & & $<0.001$ & \\
\hline
\end{tabular}

Bold denotes statistically significant result

a 4-point sleep disturbance scale: (1) I am able to sleep without difficulty most of the time; (2) My sleep is interrupted some of the time, but I am usually able to go back to sleep without difficulty; (3) My sleep is interrupted most nights, but I am usually able to go back to sleep without difficulty; (4) I sleep in short bursts only. I am awake most of the night

Model 1: Univariable analysis

Model 2: Adjusted for fixed factors (age, sex, body mass index, physical activity, comorbidities, pain medications, ever smoking, emotional problems, employment, and education level)

$R R$ relative risk, $C I$ confidence interval

Table 4 Relationship between persistent sleep disturbance and persistent knee pain and multisite pain in entire cohort

\begin{tabular}{|c|c|c|c|c|}
\hline & \multicolumn{2}{|c|}{ Model 1} & \multicolumn{2}{|c|}{ Model 2} \\
\hline & $\mathbf{R R}$ & 95\% CI & $\mathbf{R R}$ & 95\% CI \\
\hline \multicolumn{5}{|l|}{ Persistent knee pain } \\
\hline Persistent sleep disturbance & 2.13 & $1.41,3.22$ & 1.90 & $1.26,2.87$ \\
\hline \multicolumn{5}{|l|}{ Persistent multisite pain } \\
\hline Persistent sleep disturbance & 1.56 & $1.30,1.88$ & 1.29 & $1.07,1.56$ \\
\hline \multicolumn{5}{|l|}{ Persistent sleep disturbance } \\
\hline Persistent knee pain & 2.28 & $1.43,3.63$ & 1.99 & $1.23,3.24$ \\
\hline Persistent multisite pain & 2.55 & $1.55,4.19$ & 2.71 & $1.59,4.62$ \\
\hline
\end{tabular}

Bold denotes statistically significant result

Model 1: Univariable analysis

Model 2: Adjusted for age, sex, body mass index, physical activity, comorbidities, pain medications, ever smoking, emotional problems, employment, and education level

$R R$ relative risk, $C I$ confidence interval

6 months in patients with knee OA and insomnia [33]. The finding that participants having sleep disturbance were more likely to have more painful sites is partially in line with previous studies on widespread pain conducted in the general population with a follow-up of up to 18 years, showing that sleep problems were associated with new onset of widespread pain [18, 19, 34, 35] and resolution of chronic widespread pain [20]. To date, very few studies have investigated the associations between sleep disturbance and NPS; our finding is 
Table 5 Relationship between persistent sleep disturbance and persistent knee pain and multisite pain among those with radiographic knee osteoarthritis

\begin{tabular}{|c|c|c|c|c|}
\hline & \multicolumn{2}{|c|}{ Model 1} & \multicolumn{2}{|c|}{ Model 2} \\
\hline & $\overline{R R}$ & $95 \% \mathrm{CI}$ & $\overline{\mathbf{R R}}$ & $95 \% \mathrm{CI}$ \\
\hline \multicolumn{5}{|l|}{ Persistent knee pain } \\
\hline Persistent sleep disturbance & 2.12 & $1.27,3.54$ & 1.64 & $0.98,2.76$ \\
\hline \multicolumn{5}{|l|}{ Persistent multisite pain } \\
\hline Persistent sleep disturbance & 1.36 & $1.08,1.71$ & 1.21 & $1.00,1.47$ \\
\hline \multicolumn{5}{|l|}{ Persistent sleep disturbance } \\
\hline Persistent knee pain & 2.24 & $1.27,3.95$ & 1.69 & $0.93,3.06$ \\
\hline Persistent multisite pain & 1.98 & $1.07,3.64$ & 1.80 & $0.95,3.42$ \\
\hline
\end{tabular}

Bold denotes statistically significant result

Model 1: Univariable analysis

Model 2: Adjusted for age, sex, body mass index, physical activity, comorbidities, pain medications, ever smoking, emotional problems, employment, and education level

$R R$ relative risk, $C I$ confidence interval

consistent with a 2-year follow-up study, which reported that sleep disturbance was associated with greater odds of increased NPS [36]. Our study, combined with these prospective studies, indicates a link between sleep disturbance and greater pain intensity and wider distribution.

The current study assessed the temporal relationships between sleep disturbance and pain and found that interactions of sleep disturbance with time on pain intensity and NPS were not statistically significant. This suggests that the effect of sleep disturbance on pain did not change over time. One possible explanation is that pain intensity and NPS are relatively stable over time. This is supported by our results that the mean overall pain score and NPS, as well as pain across sleep disturbance groups, were maintained over time, although sleep disturbance and pain are correlated at each assessment point. Supporting this, previous research including our own has shown stable pain intensity trajectories without substantial pain improvement or worsening over time [37-41]; and the pattern of reporting NPS was also found to be stable over a 14 -year period [32].
Our study findings that persistent sleep disturbance was associated with persistent knee pain and MSP are in part agreement with previous studies [15, 42, 43]. One study with a 60-month follow-up reported that sleep quality was associated with prevalent consistent frequent knee pain among participants with or at high risk of knee $\mathrm{OA}$ [42]. In an 11-year prospective study, Mundal et al. found that poor sleep predicted persistent chronic widespread pain in a Norwegian general population [43]. Also, a recent meta-analysis concluded that persistence of sleep problems is an important contributor to worse physical health over time [15]. Although our study is not comparable with these studies, these findings reflect that persistent sleep disturbance plays a critical role in pain and have shed light on the long-term impact of sleep pattern on persistent pain. Further, a reverse association appears to support a negative impact of persistent pain on sleep disturbance. These findings highlight that the impact of sleep problems on pain should be considered in developing pain therapeutic strategies and vice versa. There was no interaction with ROA, suggesting that this association 
is not modified by the presence or absence of ROA.

Although the mechanisms involved in the sleep-pain link are not fully understood, there is some evidence suggesting that neurobiological and inflammatory mechanisms may underlie the association between sleep and pain $[16,17]$. Prior experimental sleep deprivation studies have pointed to hyperalgesic effects related to experimental disruption of sleep continuity. A recent study reported that chronic insufficient sleep may lead to alterations of pain processing related to habituation and sensitization of cold pain, which, in turn, may contribute to an increased risk of developing chronic pain conditions [44]. Further, pain intensity increased with number of days of sleep restriction [45]. Inflammation is fundamental in alteration of pain modulation and pain processing $[46,47]$. One meta-analysis including 72 cohort and experimental sleep deprivation studies suggested that sleep disturbance was associated with an elevated level of inflammatory markers [i.e. C-reactive protein (CRP), interleukin-6 (IL6)] [47]. Interestingly, patients with persistent insomnia appeared to have a higher CRP as compared with those with intermittent or never insomnia [48]. These mechanisms may explain a dose-response relationship between sleep disturbance and pain observed in the current study, as well as the impact of persistent sleep disturbance on persistent pain where abnormalities of pain processing are involved.

More than two assessments of sleep and quantitative pain, long-term observation period, and evaluating ROA-specific sleep-pain relationship are the strengths of this study. However, several limitations need to be acknowledged. One of the major limitations is that sleep was assessed by one item from a selfreported questionnaire. This subjective questionnaire does not prospectively record the quality of sleep and may lead to recall biases. Second, participants with severe sleep disturbance may seek sleep treatments (i.e. CBT or pharmacological interventions). However, these data were not collected in this study, which may have weakened the impact of sleep disturbance on pain. Third, NPS was measured by a yes/no questionnaire which cannot allow for examinations of whether sleep disturbance was associated with pain intensity in sites other than the knees.

\section{CONCLUSION}

Sleep disturbance was independently associated with greater pain severity and NPS in a dose-response manner. A reciprocal relationship between persistent sleep disturbance and persistent pain suggests treating either problem could help the other.

\section{ACKNOWLEDGEMENTS}

We thank the study participants for volunteering for the study.

Funding. This study was supported by the National Health and Medical Research Council (NHMRC) project Grant of Australia (302204). FP is funded by the NHMRC Early Career Fellowship; JT is funded by the National Heart Foundation Fellowship; GJ is funded by NHMRC Practitioner Fellowship. No Rapid Service Fee was received by the journal for the publication of this article.

Authorship. All named authors meet the International Committee of Medical Journal Editors (ICMJE) criteria for authorship for this article, take responsibility for the integrity of the work as a whole, and have given their approval for this version to be published.

Disclosures. Feng Pan, Jing Tian, Flavia Cicuttini and Graeme Jones have nothing to disclose.

Compliance with Ethics Guidelines. This study was approved by the Southern Tasmanian Health and Medical Human Research Ethics Committee (Ref. no. H0006488), and written informed consent of all participants was obtained. This study was performed in accordance with the Helsinki Declaration of 1964 and its later amendments. 
Data Availability. The datasets generated during and/or analysed during the current study are available from the corresponding author on reasonable request.

Open Access. This article is licensed under a Creative Commons Attribution-NonCommercial 4.0 International License, which permits any non-commercial use, sharing, adaptation, distribution and reproduction in any medium or format, as long as you give appropriate credit to the original author(s) and the source, provide a link to the Creative Commons licence, and indicate if changes were made. The images or other third party material in this article are included in the article's Creative Commons licence, unless indicated otherwise in a credit line to the material. If material is not included in the article's Creative Commons licence and your intended use is not permitted by statutory regulation or exceeds the permitted use, you will need to obtain permission directly from the copyright holder. To view a copy of this licence, visit http://creativecommons.org/licenses/by$\mathrm{nc} / 4.0 /$.

\section{REFERENCES}

1. Léger D, Poursain B, Neubauer D, Uchiyama M. An international survey of sleeping problems in the general population. Curr Med Res Opin. 2008;24: 307-17.

2. Adams RJ, Appleton SL, Taylor AW, et al. Sleep health of Australian adults in 2016: results of the 2016 Sleep Health Foundation national survey. Sleep Health. 2017;3:35-42.

3. Stranges S, Tigbe W, Gomez-Olive FX, Thorogood M, Kandala NB. Sleep problems: an emerging global epidemic? Findings from the INDEPTH WHO-SAGE study among more than 40,000 older adults from 8 countries across Africa and Asia. Sleep. 2012;35: 1173-81.

4. Yin J, Jin X, Shan Z, et al. Relationship of sleep duration with all-cause mortality and cardiovascular events: a systematic review and dose-response meta-analysis of prospective cohort studies. J Am Heart Assoc. 2017;6:e005947.

5. Stickley A, Leinsalu M, DeVylder JE, Inoue Y, Koyanagi A. Sleep problems and depression among
237,023 community-dwelling adults in 46 low- and middle-income countries. Sci Rep. 2019;9:12011.

6. Cimmino MA, Ferrone C, Cutolo M. Epidemiology of chronic musculoskeletal pain. Best Pract Res Clin Rheumatol. 2011;25:173-83.

7. McBeth J, Jones K. Epidemiology of chronic musculoskeletal pain. Best Pract Res Clin Rheumatol. 2007;21:403-25.

8. Fayaz A, Croft P, Langford RM, Donaldson LJ, Jones GT. Prevalence of chronic pain in the UK: a systematic review and meta-analysis of population studies. BMJ Open. 2016;6:e010364.

9. Cross M, Smith E, Hoy D, et al. The global burden of hip and knee osteoarthritis: estimates from the global burden of disease 2010 study. Ann Rheum Dis. 2014;73:1323-30.

10. Smith MT, Haythornthwaite JA. How do sleep disturbance and chronic pain inter-relate? Insights from the longitudinal and cognitive-behavioral clinical trials literature. Sleep Med Rev. 2004;8: 119-32.

11. Frohnhofen H. Pain and sleep: a bidirectional relationship. Z Gerontol Geriatr. 2018;51:871-4.

12. Ho KKN, Ferreira PH, Pinheiro MB, et al. Sleep interventions for osteoarthritis and spinal pain: a systematic review and meta-analysis of randomized controlled trials. Osteoarthr Cartil. 2019;27: 196-218.

13. Andersen ML, Araujo P, Frange C, Tufik S. Sleep disturbance and pain: a tale of two common problems. Chest. 2018;154:1249-59.

14. Finan PH, Goodin BR, Smith MT. The association of sleep and pain: an update and a path forward. J Pain. 2013;14:1539-52.

15. Afolalu EF, Ramlee F, Tang NKY. Effects of sleep changes on pain-related health outcomes in the general population: a systematic review of longitudinal studies with exploratory meta-analysis. Sleep Med Rev. 2018;39:82-97.

16. Haack M, Simpson N, Sethna N, Kaur S, Mullington J. Sleep deficiency and chronic pain: potential underlying mechanisms and clinical implications. Neuropsychopharmacology. 2020;45:205-16.

17. Herrero Babiloni A, De Koninck BP, Beetz G, De Beaumont L, Martel MO, Lavigne GJ. Sleep and pain: recent insights, mechanisms, and future directions in the investigation of this relationship. J Neural Transm (Vienna). 2020;127:647-60. 
18. McBeth J, Lacey RJ, Wilkie R. Predictors of newonset widespread pain in older adults: results from a population-based prospective cohort study in the UK. Arthritis Rheumatol. 2014;66:757-67.

19. Gupta A, Silman AJ, Ray D, et al. The role of psychosocial factors in predicting the onset of chronic widespread pain: results from a prospective population-based study. Rheumatology (Oxford). 2007;46:666-71.

20. Davies KA, Macfarlane GJ, Nicholl BI, et al. Restorative sleep predicts the resolution of chronic widespread pain: results from the EPIFUND study. Rheumatology (Oxford). 2008;47:1809-13.

21. McBeth J, Wilkie R, Bedson J, Chew-Graham C, Lacey RJ. Sleep disturbance and chronic widespread pain. Curr Rheumatol Rep. 2015;17:469.

22. Lacey RJ, Belcher J, Rathod T, Wilkie R, Thomas E, McBeth J. Pain at multiple body sites and healthrelated quality of life in older adults: results from the North Staffordshire Osteoarthritis Project. Rheumatology (Oxford). 2014;53:2071-9.

23. Nicholl BI, Mackay D, Cullen B, et al. Chronic multisite pain in major depression and bipolar disorder: cross-sectional study of 149,611 participants in UK Biobank. BMC Psychiatry. 2014;14:350.

24. Westoby CJ, Mallen CD, Thomas E. Cognitive complaints in a general population of older adults: prevalence, association with pain and the influence of concurrent affective disorders. Eur J Pain. 2009;13:970-6.

25. Pan F, Byrne KS, Ramakrishnan R, Ferreira M, Dwyer T, Jones G. Association between musculoskeletal pain at multiple sites and objectively measured physical activity and work capacity: results from UK Biobank study. J Sci Med Sport. 2019;22:444-9.

26. Hawthorne G, Richardson J, Osborne R. The Assessment of Quality of Life (AQoL) instrument: a psychometric measure of health-related quality of life. Qual Life Res. 1999;8:209-24.

27. Carnes D, Parsons S, Ashby D, et al. Chronic musculoskeletal pain rarely presents in a single body site: results from a UK population study. Rheumatology (Oxford). 2007;46:1168-70.

28. Altman RD, Hochberg M, Murphy WA Jr, Wolfe F, Lequesne M. Atlas of individual radiographic features in osteoarthritis. Osteoarthr Cartil. 1995;3(Suppl A):3-70.

29. Ware J, Kosinski M, Dewey J, Gandek B. A manual for users of the SF-8 Health Survey. Lincoln: Quality Metric; 2001.
30. Dore DA, Winzenberg TM, Ding C, et al. The association between objectively measured physical activity and knee structural change using MRI. Ann Rheum Dis. 2013;72:1170-5.

31. Seaman SR, White IR. Review of inverse probability weighting for dealing with missing data. Stat Methods Med Res. 2013;22:278-95.

32. Kamaleri Y, Natvig B, Ihlebaek CM, Benth JS, Bruusgaard D. Change in the number of musculoskeletal pain sites: a 14-year prospective study. Pain. 2009;141:25-30.

33. Smith MT, Finan PH, Buenaver LF, et al. Cognitivebehavioral therapy for insomnia in knee osteoarthritis: a randomized, double-blind, active placebo-controlled clinical trial. Arthritis Rheumatol. 2015;67:1221-33.

34. Davies KA, Silman AJ, Macfarlane GJ, et al. The association between neighbourhood socio-economic status and the onset of chronic widespread pain: results from the EPIFUND study. Eur J Pain. 2009;13:635-40.

35. Aili K, Andersson M, Bremander A, Haglund E, Larsson I, Bergman S. Sleep problems and fatigue as predictors for the onset of chronic widespread pain over a 5- and 18-year perspective. BMC Musculoskelet Disord. 2018;19:390.

36. Christensen JO, Johansen S, Knardahl S. Psychological predictors of change in the number of musculoskeletal pain sites among Norwegian employees: a prospective study. BMC Musculoskelet Disord. 2017;18:140.

37. Collins JE, Katz JN, Dervan EE, Losina E. Trajectories and risk profiles of pain in persons with radiographic, symptomatic knee osteoarthritis: data from the osteoarthritis initiative. Osteoarthr Cartil. 2014;22:622-30.

38. Nicholls E, Thomas E, van der Windt DA, Croft PR, Peat G. Pain trajectory groups in persons with, or at high risk of, knee osteoarthritis: findings from the Knee Clinical Assessment Study and the Osteoarthritis Initiative. Osteoarthr Cartil. 2014;22: 2041-50.

39. Bastick AN, Wesseling J, Damen J, et al. Defining knee pain trajectories in early symptomatic knee osteoarthritis in primary care: 5-year results from a nationwide prospective cohort study (CHECK). Br J Gen Pract. 2016;66:e32-9.

40. Dai Z, Lu N, Niu J, Felson DT, Zhang Y. Dietary fiber intake in relation to knee pain trajectory. Arthritis Care Res (Hoboken). 2017;69:1331-9. 
41. Pan F, Tian J, Aitken D, Cicuttini F, Jones G. Predictors of pain severity trajectory in older adults: a 10.7-year follow-up study. Osteoarthr Cartil. 2018;26:1619-26.

42. Dai Z, Neogi T, Brown C, et al. Sleep quality is related to worsening knee pain in those with widespread pain: the Multicenter Osteoarthritis Study. J Rheumatol. 2019;47:1019.

43. Mundal I, Grawe RW, Bjorngaard JH, Linaker OM, Fors EA. Prevalence and long-term predictors of persistent chronic widespread pain in the general population in an 11-year prospective study: the HUNT study. BMC Musculoskelet Disord. 2014;15: 213.

44. Simpson NS, Scott-Sutherland J, Gautam S, Sethna N, Haack M. Chronic exposure to insufficient sleep alters processes of pain habituation and sensitization. Pain. 2018;159:33-40.
45. Haack M, Mullington JM. Sustained sleep restriction reduces emotional and physical well-being. Pain. 2005;119:56-64.

46. De Jongh RF, Vissers KC, Meert TF, Booij LH, De Deyne CS, Heylen RJ. The role of interleukin-6 in nociception and pain. Anesth Analg. 2003;96: 1096-103.

47. Irwin MR, Olmstead R, Carroll JE. Sleep disturbance, sleep duration, and inflammation: a systematic review and meta-analysis of cohort studies and experimental sleep deprivation. Biol Psychiatry. 2016;80:40-52.

48. Parthasarathy S, Vasquez MM, Halonen M, et al. Persistent insomnia is associated with mortality risk. Am J Med. 2015;128(268-75):e2. 